\title{
A NEW FORM OF THE SIMPLEST PROBLEM OF THE
}

\section{CALCULUS OF VARIATIONS*}

\author{
BY \\ GILBERT AMES BLISS
}

The simplest problem of the calculus of variations in the plane has been studied in two different forms. In the earlier one the curves considered are represented by equations

$$
y=y(x),
$$

where $y(x)$ is a single-valued function, and the integral to be minimized is

$$
I=\int f\left(x, y, y^{\prime}\right) d x .
$$

In the later form, which seems first to have been studied in detail by WEIERSTRASS, the curves are taken in parametric representation

$$
C: \quad x=\phi(t), \quad y=\psi(t),
$$

and the integral in the form

$$
I=\int F\left(x, y, x_{t}, y_{t}\right) d t
$$

where the function $F$ is positively homogeneous in the derivatives $x_{t}$ and $y_{t}$. Both methods are open to objections, the former because it is inconvenient to apply to curves for which the function $y(x)$ is not single valued or for which the slope becomes infinite. In fact, no complete discussion has been attempted for the problem of minimizing the integral (1) without restricting the functions $y(x)$ to be single-valued and to have a finite derivative. The second form of the problem is complicated by the fact that in all the calculations involved the condition of homogeneity

$$
F\left(x, y, \kappa x_{t}, \kappa y_{t}\right)=\kappa F\left(x, y, x_{t}, y_{t}\right)
$$

must be kept in mind and frequently applied.

In a paper entitled $A$ generalization of the notion of angle $\dagger$ the writer has introduced a third form of the problem, in which the integral involves only invariants of the curve (2) under change of parameter-representation. The integral there given is

* Presented to the Society April 27, 1907. Received for publication April 8, 1907.

†Transactions of the American Mathematical Society, vol. 7 (1906), p. 184. 


$$
I=\int f(x, y, \tau) d s,
$$

where $s$ is the length of arc along the curve (2), and $\tau$ is the angle made by the curve with the $x$-axis, which is defined by the equations

$$
\cos \tau=\frac{x_{t}}{\sqrt{x_{t}^{2}+y_{t}^{2}}}, \quad \sin \tau=\frac{y_{t}}{\sqrt{x_{t}^{2}+y_{t}^{2}}} .
$$

The integral (3) may be easily put into the form (4), for on account of the homogeneity of $F$,

$$
F\left(x, y, x_{t}, y_{t}\right)=F(x, y, \cos \tau, \sin \tau) \sqrt{x_{t}^{2}+y_{t}^{2}} .
$$

But in (4) the function $f(x, y, \tau)$ need not be periodic as is the case with the right member of the last equation. Besides this generalization, the advantages of having the integral in the form (4) are that one can use the parametric representation for curves and at the same time avoid the troublesome homogeneity condition connected with the Weierstrassian form of the problem. It must be admitted that the derivation of the important formulas is not in general as simple as is the case for the integral (1), but otherwise the advantages of both of the older methods seem to be retained. The new form of the problem is especially advantageous in connection with the inverse problem of the calculus of variations and its application to geometry as made by $\mathrm{H}_{A M E L},{ }^{*}$ and in determining the invariants of the problem under point transformations.

In the paper by the writer referred to above only the Euler equation and the equations defining transversality, which were necessary in finding the generalization of angle, were developed. It is proposed here to complete the theory of the problem connected with the integral (4) by deriving the forms of the first and second variations and the functions which are commonly used in proving the necessary conditions in the calculus of variations. The derivation of the conditions themselves can be made as is usual in the books on the subject. $\dagger$

\section{§1. The first variation.}

Suppose then that the integral to be studied is

where

$$
I=\int f(x, y, \tau) \sqrt{x_{t}^{2}+y_{t}^{2}} d t
$$

$$
\sqrt{x_{t}^{2}+y_{t}^{2}}=\frac{d s}{d t}
$$

* Über die Geometrieen in denen die Geraden die Kürzesten sind, Math ematische A nnalen, vol. 57 (1903), p. 231.

† See especially BoLza, Lectures on the Calculus of Variations. 
and where the function $f(x, y, \tau)$ is of class $C^{\mathrm{iv} *}$ in a region $R$ of $(x, y, \tau)$-points having an interior. The curves considered will all be represented in the parametric representation (2) where $\phi$ and $\psi$ are functions of class $C^{\text {iv }}$ and define values $(x, y, \tau)$ lying interior to the region $R$ for all values of $t$ in the interval $t_{0} \leqq t \leqq t_{1}$. As the value of $\tau$ is to be well-defined for every value of $t$, it is evident that the expression $x_{t}^{2}+y_{t}^{2}$ must be everywhere different from zero, and the curve will therefore be supposed to have no singular points.

If the integral $I$ is taken along one of the family of curves

$$
C_{a}^{\gamma}: \quad x=\phi(t, \alpha), \quad y=\psi(t, \alpha)
$$

where $\phi(t, \alpha)$ and $\psi(t, \alpha)$ are of class $C^{\mathrm{iv}}$ in the region

$$
t_{0} \leqq t \leqq t_{1}, \quad|\alpha| \leqq k
$$

and for $\alpha=0$ define the curve (2), then the value of $I$ becomes a function $I(\alpha)$ which for $\alpha=0$ reduces to the value $I$ taken along $C$. The first derivative is

$$
\frac{d I}{d \alpha}=\int_{t_{0}}^{t_{1}}\left\{f_{x} x_{a}+f_{y} y_{a}+f_{\tau} \tau_{a}+f \frac{x_{t} x_{a t}+y_{t} y_{a t}}{x_{t}^{2}+y_{t}^{2}}\right\} \sqrt{x_{t}^{2}+y_{t}^{2}} d t .
$$

In this and following formulas literal subscripts denote partial differentiation, except the subscript $s$ which means that there has first been a partial differentiation with respect to $t$ and then a division by $s_{t}=\sqrt{x_{t}^{2}+y_{t}^{2}}$.

After substitution of the value of $\tau_{a}$ calculated from equations (5) and (6), the last equation becomes

$$
\frac{d I}{d \alpha}=\int_{s_{0}}^{s_{1}}\left\{f_{x} x_{a}+f_{y} y_{\alpha}+\left(f \cos \tau-f_{\tau} \sin \tau\right) x_{a s}+\left(f \sin \tau+f_{\tau} \cos \tau\right) y_{a s}\right\} d s,
$$

or by the usual partial integration of the calculus of variations,

$\left.\frac{d I}{d \alpha}=\int_{s_{0}}^{s_{1}}\left\{P x_{a}+Q y_{a}\right\} d s+\left[\left(f \cos \tau-f_{\tau} \sin \tau\right) x_{a}+\left(f \sin \tau+f_{\tau} \cos \tau\right) y_{a}\right]\right]_{s_{0}}^{s_{1}}$, where

$$
P=f_{x}-\frac{d}{d s}\left(f \cos \tau-f_{\tau} \sin \tau\right), \quad Q=f_{y}-\frac{d}{d s}\left(f \sin \tau+f_{\tau} \cos \tau\right) .
$$

By an easy calculation $P$ and $Q$ are found to satisfy the relation

$$
P \cos \tau+Q \sin \tau=0,
$$

so that a new expression $T$ can be introduced by means of the equations

$$
P=T \sin \tau, \quad Q=-T \cos \tau .
$$

* A function of class $C^{(n)}$ is by definition one which is itself continuous and has continuous derivatives up to and including those of the $n$th order. The assumptions on $f(x, y, \tau)$ and the curve (2) insure the existence of all the derivatives used in the following developments. 
The final form of the first derivative is then

(7) $\frac{d I}{d \alpha}=\int_{s_{0}}^{s_{1}} w T d s+\left[\left(f \cos \tau-f_{\tau} \sin \tau\right) x_{a}+\left(f \sin \tau+f_{\tau} \cos \tau\right) y_{a}\right]_{s_{1}}^{s_{0}}$,

where

$$
w=x_{a} \sin \tau-y_{a} \cos \tau,
$$

(9) $T\left(x, y, \tau, \tau_{s}\right)=f_{x} \sin \tau-f_{y} \cos \tau+f_{x \tau} \cos \tau+f_{y \tau} \sin \tau+\left(f+f_{\tau \tau}\right) \tau_{s}$.

In case the curves (6) all pass through the two end-points 0 and 1 of the arc $C$, one sees readily by putting $t=t_{0}$ or $t=t_{1}$ in equations (6) and differentiating for $\alpha$, that $x_{a}, y_{a}$ both vanish at the points 0 and 1 , so that for $a$ set of curves (6) all passing through two fixed points 0 and 1 the derivative $d I / d \alpha$ has the value

$$
\frac{d I}{d \alpha}=\int_{s_{0}}^{s_{1}} w T d s,
$$

where $w$ and $T$ are the expressions in (8) and (9) above.

The function $w$ depends upon the family of curves (6). But if $w$ is given in advance, a family can always be found including the arc $C$ for $\alpha=0$ and having the given value $w$. It is only necessary to take curves in the form

$$
x=\phi(t)+\alpha \xi, \quad y=\psi(t)+a \eta,
$$

where $\xi$ and $\eta$ are to be determined by the equations

$$
\begin{aligned}
& \xi \sin \tau-\eta \cos \tau=w, \\
& \xi \cos \tau+\eta \sin \tau=0 .
\end{aligned}
$$

In particular, if $w$ vanishes for $t=t_{0}$ and $t=t_{1}$, the curves (10) will all pass through two fixed points.

By methods well-known in the calculus of variations it follows then that any curve which joins two given fixed points and minimizes the integral I must be a solution of Euler's differential equation

$$
T\left(x, y, \tau, \tau_{s}\right)=0 .
$$

\section{§2. The second variation.}

For the case when the end-points are fixed the second derivative $d^{2} I / d \alpha^{2}$ can be calculated from the expression

$$
\frac{d I}{d \alpha}=\int_{t_{0}}^{t_{1}} T \cdot w \sqrt{x_{t}^{2}+y_{t}^{2}} d t .
$$

It is seen readily that the derivative of the integrand is

$$
\left.w\left\{T_{x} x_{a}+T_{. .} y_{a}+T_{\tau} \tau_{a}+\left(f+f_{\tau \tau}\right) \tau_{s a}\right\} \sqrt{x_{t}^{2}+y_{t}^{2}}+T_{\overline{\partial \alpha}} \frac{\partial}{w} \bar{v} \overline{x_{t}^{2}+y_{t}^{2}}\right) .
$$


The value of this second derivative is desired for $\alpha=0$, that is, along the curve $C$. If $C$ satisfies the first necessary condition for a minimum, $T=0$, the term with the factor $T$ disappears. Furthermore from (9) and (5)

$$
\begin{gathered}
T_{\tau}=f_{s}+f_{\tau \tau}, \quad \tau_{a}=\left(x_{a} \cos \tau+y_{a} \sin \tau\right) \tau_{s}-w_{s}, \\
\tau_{s a}=\frac{\partial}{\partial \alpha} \frac{\tau_{t}}{\sqrt{x_{t}^{2}+y_{t}^{2}}}=\left(x_{a} \cos \tau+y_{a} \sin \tau\right) \tau_{s}-w_{s s}-\tau_{s}^{2} w,
\end{gathered}
$$

so that for $\alpha=0$ the expression (12) becomes

$$
w\left\{\left(T_{x}+\cos \tau \frac{d}{d s} f_{1} \tau_{s}\right) x_{a}+\left(T_{y}+\sin \tau \frac{d}{d s} f_{1} \tau_{s}\right) y_{\alpha}-f_{1} \tau_{s}^{2} w-\frac{d}{d s} f_{1} w_{s}\right\}
$$

where

But along the curve $C$

$$
f_{1}=f+f_{\tau \tau}
$$

$$
\left(T_{x}+\cos \tau \frac{d}{d s} f_{1} \tau_{\bullet}\right) \cos \tau+\left(T_{y}+\sin \tau \frac{d}{d s} f_{1} \tau_{\bullet}\right) \sin \tau=T_{s}=0 .
$$

When this equation and

$\left(T_{x}+\cos \tau \frac{d}{d s} f_{1} \tau_{c}\right) \sin \tau-\left(T_{y}+\sin \tau \frac{d}{d s} f_{1} \tau_{s}\right) \cos \tau=T_{x} \sin \tau-T_{y} \cos \tau$

are solved for the parentheses and the results substituted in (14), one finds without difficulty

$$
\left.\frac{d^{2} I}{d \alpha^{2}}\right|^{a=0}=\int_{s_{0}}^{s_{1}} w\left(f_{2} w-\frac{d}{d s} f_{1} w_{\diamond}\right) d s=\int_{s_{0}}^{s_{1}}\left\{f_{1} w_{s}^{2}+f_{2} w^{2}\right\} d s,
$$

where

$$
f_{2}\left(x, y, \tau, \tau_{\lrcorner}\right)=T_{x} \sin \tau-T_{y} \cos \tau-f_{1} \tau_{a}^{2} .
$$

The latter part of (15) is derived from the former by a simple partial integration, the terms outside the integral disappearing because for fixed end-points $w$ vanishes at $s=s_{0}$ and $s=s_{1}$. These, however, are the well-known forms for the second variation, a factor $\alpha^{2} / 2$ only being omitted, from which one derives the two following conditions.

LEGENDRE's NECESSARY CONDITION. The function $f_{1}=f+f_{\tau \tau}$ must be $\geqq 0$ for a minimum, or $\leqq 0$ for a maximum, at every point of a minimizing $\operatorname{arc} C$.

JACOBI's NECESSARY CONDITION. If the arc $C$ satisfies Euler's equation and if furthermore $f_{1} \neq 0$ along $C$, then a further necessary condition for a minimum or maximum is that no integral $w$ of Jacobi's equation,

$$
f_{2} w-\frac{d}{d s} f_{1} w_{s}=0,
$$

exists which vanishes more than once in the interval $s_{0} \leqq s<s_{1}$, the arguments of $f_{2}\left(x, y, \tau, \tau_{s}\right)$ and $f_{1}(x, y, \tau)$ in this equation being those corresponding to the arc $C$. 
§ 3. Hilbert's invariant integral and the E-function of Weierstrass.

As soon as it is known that the arc $C$ is a solution of Euler's equation along which $f_{1} \neq 0$ and which satisfies Jacobi's condition, the existence theorems for differential equations enable one to imbed $C$ in a field of solutions of Euler's equation. In fact, if a point 2 is taken near enough to 0 on $C$ and having the order 201 with the points 0 and 1 , then the solutions of (11) which pass through the point 2 will be a one-parameter family of curves which simply cover a portion of the plane in the neighborhood of the arc $C .^{*}$ Their equations can be found in the form

$$
x=\phi(t, \alpha), \quad y=\psi(t, \alpha) \quad(0 \leqq t \leqq T,|\alpha| \leqq k),
$$

where the value $t=0$ corresponds to the point 2 on each curve, and where for $\alpha=0$ the arc $C$ itself is represented. Under the assumptions originally made upon the function $f^{\prime}(x, y, \tau)$ the functions $\phi$ and $\psi$ will be of class $C^{\text {iv }}$.

In the field surrounding $C$ the equations (16) are uniquely solvable for $t$ and $\alpha$ in terms of $x$ and $y$,

$$
t=t(x, y), \quad \alpha=\alpha(x, y),
$$

and by implicit function theory these functions will be of class $C^{\prime}$ at least.

HiLberT's invariant integral may be derived from the integral

$$
I=\int_{0}^{t} f(x, y, \tau) d s
$$

taken along any one of the solutions (16) from the point $2(t=0)$ to a point corresponding to the value $t$. This integral is a function of $t$ and $\alpha$, or by means of equations (17), a function of $x$ and $y$ in the field. If one takes the integral

over any path

$$
I^{*}=\int_{u_{3}}^{u_{4}}\left\{\frac{\partial I}{\partial x} x_{u}+\frac{\partial I}{\partial y} y_{u}\right\} d u
$$

$$
\bar{C}: \quad x=x(u), \quad y=y(u),
$$

in the field joining two given points 3,4 , it is evident that its value will always be the same however the path is chosen. The integral (18) is indeed HilberT's invariant integral. It is desirable, however, to find more definitely the expres. sions for $\partial I / \partial x$ and $\partial I / \partial y$. By steps like those of $\S 1$ it is seen that

$$
\begin{aligned}
& \frac{\partial I}{\partial t}=f(x, y, \tau) \sqrt{x_{t}^{2}+y_{t}^{2}}, \\
& \frac{\partial I}{\partial \alpha}=\left(f \cos \tau-f_{\tau} \sin \tau\right) x_{a}+\left(f \sin \tau+f_{\tau} \cos \tau\right) y_{\alpha} .
\end{aligned}
$$

* In the case when the function $f(x, y, \tau)$ is periodic with the period $2 \pi$ in $\tau$ the family of solutions through the point 0 has the same property. See BLIss, The construction of a field of extremals about a given point, Bulletin of the a $_{-}$merican Mathematioal Society, vol. 13 (1907), p. 321 . 
Since the curves (16) are all solutions of Euler's equation the expression $T$ vanishes identically, while $x_{\alpha}$ and $y_{\alpha}$ vanish at the point 0 , so that in equation (17) only the terms outside the integral sign at the point $t$ are left. It follows therefore that

$\frac{\partial I}{\partial x}=f(x, y, \tau) \sqrt{x_{t}^{2}+y_{\imath}^{2}} \frac{\partial t}{\partial x}+\left[\left(f \cos \tau-f_{\tau} \sin \tau\right) x_{a}+\left(f \sin \tau+f_{\tau} \cos \tau\right) y_{a}\right] \frac{\partial \alpha}{\partial x}$,

with a similar equation for the $y$-derivative. The integrand of HilberT's invariant integral (18) is evidently

$f(x, y, \tau) \sqrt{x_{t}^{2}+y_{t}^{2}} \frac{d t}{d u}+\left[\left(f \cos \tau-f_{\tau} \sin \tau\right) x_{\alpha}+\left(f \sin \tau+f_{\tau} \cos \tau\right) y_{a}\right] \frac{d \alpha}{d u}$,

which can be easily changed into the form

since

$$
\left(f \cos \tau-f_{\tau} \sin \tau\right) x_{u}+\left(f \sin \tau+f_{\tau} \cos \tau\right) y_{u}
$$

$$
\begin{gathered}
\sqrt{x_{t}^{2}+y_{t}^{2}}=x_{t} \cos \tau+y_{t} \sin \tau, \\
x_{t} \frac{d t}{d u}+x_{a} \frac{d \alpha}{d u}=x_{u}, \quad y_{t} \frac{d t}{d u}+y_{a} \frac{d \alpha}{d u}=y_{u} .
\end{gathered}
$$

If an angle $\sigma$ is defined by the equations

$$
\cos \sigma=\frac{x_{u}}{\sqrt{x_{u}^{2}+y_{u}^{2}}}, \quad \sin \sigma=\frac{y_{u}}{\sqrt{x_{u}^{2}+y_{u}^{2}}},
$$

Hilbert's integral may be written in the form

$$
I^{*}=\int\left\{f^{\prime} \cos (\sigma-\tau)+f_{\tau} \sin (\sigma-\tau)\right\} \sqrt{x_{u}^{2}+y_{u}^{2}} d u,
$$

where $\sigma$ and $\tau$ are the direction-angles of the arbitrarily chosen curve (19) and one of the solutions (16) of Euler's equation, respectively, at their point of intersection in the field. This integral has the fundamental properties that its value taken between any two points of the field is independent of the path over which it is taken, and on one of the solutions (16) of Euler's equation it has the same value as the original integral $I$.

The latter property follows at once, since along an extremal $\sigma=\tau$.

The WeIERSTRASS $E$-function is the function

$$
E(x, y, \sigma, \tau)=f \cos (\sigma-\tau)+f_{\tau} \sin (\sigma-\tau)-f(x, y, \sigma)
$$

occurring in the integrand of the difference

$$
I(\bar{C})-I^{*}(\bar{C})=\int E \sqrt{x_{u}^{2}+y_{u}^{2}} d u,
$$


taken along any curve $\vec{C}$ of the field. If the ends of the arc over which the integration is taken lie on the arc $C$, this difference has the value

$$
I(\bar{C})-I^{*}(\bar{C})=I(\bar{C})-I(C)
$$

on account of the properties of $I^{*}$ above mentioned.

By means of special variations WeIERstrass derived the following fourth necessary condition involving the $E$-function.*

WEIERSTRASS's NECESSARY CONDITION. If the arc $C$ is a solution of Euler's equation which minimizes the integral $I$ with respect to any curve lying in a properly chosen neighborhood of $C$ and joining its end-points, then the inequality

$$
E\left(x^{\prime}, y, \sigma, \tau\right) \geqq 0
$$

must hold for every set of values $(x, y, \tau)$ corresponding to a point on $C$ and for all values of $\sigma$. In case there is a maximum $E$ must be $\leqq 0$.

It is well known that the four necessary conditions given above do not at the same time insure a minimum or a maximum. By strengthening the inequalities involved in them, however, new sets of conditions may be derived in various ways which with the help of a field and the equations (20), (21) can be proved sufficient. For the sake of completeness one of the theorems which define sufficient conditions is given-here.

If the arc $C$ is a solution of Euler's equation along which Legendre's and Weierstrass's conditions hold in the stronger forms $f_{1}>0$ and $E>0$, and if furthermore Jacobi's condition is satisfied not only in the interval $s_{0} \leqq s<s_{1}$ but also in $s_{0} \leqq s \leqq s_{1}$, then a neighborhood of $C$ can always be found in which this arc actually minimizes the integral $I$.

\section{§4. Variable endpoints.}

In the preceding sections the formulæ derived were for the case of fixed endpoints. When a minimizing arc $C$ is sought, however, which joins a fixed point 0 with a fixed curve $D$, a new condition on the directions of $C$ and $D$ at their point of intersection must be satisfied and the $J_{A C O B I}$ condition takes on a somewhat different form. For the purpose of deriving these conditions let the representation of the family of curves (6) be chosen, as is usually done, so that their intersections with the curve $D$ all occur at the fixed parameter-value $t_{1}$. Then the curve $D$ is represented by the equations

$$
x=\phi\left(t_{1}, \alpha\right), \quad y=\psi\left(t_{1}, \alpha\right),
$$

in which $\alpha$ is to be regarded as the parameter. The integral $I$, taken over a curve of this set and regarded as a function of $\alpha$, has the first derivative

* See BolzA, Lectures on the calculus of variations, p. 138. 


$$
\frac{d I}{d \alpha}=\int_{s_{0}}^{s_{1}} w T d s+\left[\sqrt{x_{\alpha}^{2}+y_{a}^{2}}\left\{f \cos (\sigma-\tau)+f_{\tau} \sin (\sigma-\tau)\right\}\right]^{t=t_{1}},
$$

a formula derived from equation (7), in which $\sigma$ denotes the direction of the curve $D$ derived from the equations

$$
\cos \sigma=\frac{x_{a}}{\sqrt{x_{a}^{2}+y_{a}^{2}}}, \quad \sin \sigma=\frac{y_{a}}{\sqrt{x_{a}^{2}+y_{a}^{2}}},
$$

for $t=t_{1}$. The terms outside the integral in (7) vanish at the point 0 exactly as before, but not necessarily at $t=t_{1}$. If the arc $C$ is to minimize $I$ with respect to all curves joining the point 0 with the curve $D$, it must certainly minimize $I$ with respect to curves which join 0 with the intersection of $C$ and $D$, and consequently must be a solution of Euler's equation as in $\S 1$. In that case the integral part of the expression (22) vanishes for $\alpha=0$. But the rest must also vanish for $\alpha=0$, and so the following condition must be satisfied.

Condition of transversality. If the arc $C$ is a solvition of Euler's equation which minimizes the integral $I$ with respect to curves joining a fixed point 0 with a fixed curve $D$, then at its intersection with $D$ the equation

$$
f \cos (\sigma-\tau)+f_{\tau} \sin (\sigma-\tau)=0
$$

must also be satisfied, where $\sigma$ denotes the direction of $D$ and $\tau$ the direction of $C$.*

For the second derivative $d^{2} I / d \alpha^{2}$, the integral part has the form given first in equation (15), and it remains only to find the derivative of the terms in (22) which are outside the integral sign. For $\alpha=0$ the expression

$$
\left[f \cos (\sigma-\tau)+f_{\tau} \sin (\sigma-\tau)\right] \frac{\partial}{\partial \alpha} \sqrt{x_{\alpha}^{2}+y_{a}^{2}}
$$

vanishes on account of equation (23). The rest of the derivative, omitting a factor $\sqrt{x_{a}^{2}+y_{a}^{2}}$, is

$$
\begin{aligned}
& {\left[-f \sin (\sigma-\tau)+f_{\tau} \cos (\sigma-\tau)\right]\left(\sigma_{a}-\tau_{a}\right)} \\
& \quad+\left(f_{x} x_{a}+f_{y} y_{a}+f_{\tau} \tau_{a}\right) \cos (\sigma-\tau)+\left(f_{\tau x} x_{a}+f_{\tau y} y_{a}+f_{\tau \tau} \tau_{a}\right) \sin (\sigma-\tau) .
\end{aligned}
$$

In this expression, from (13) and the theory of plane curves,

$$
\tau_{a}=\sqrt{x_{a}^{2}+y_{a}^{2}} \cos (\sigma-\tau) \cdot \tau_{s}-w_{s}, \quad \sigma_{a}=\frac{\sqrt{x_{a}^{2}+y_{a}^{2}},}{r}
$$

* The equation (23) is satisfied if

$$
\cos \sigma=\frac{f \sin \tau+f_{\tau} \cos \tau}{\sqrt{f^{2}+f_{\tau}^{2}}}, \quad \sin \sigma=\frac{-f \cos \tau+f_{\tau} \sin \tau}{\sqrt{f^{2}+f_{\tau}^{2}}},
$$

as in the writer's earlier paper referred to above. 
where $r$ is the radius of curvature of the curve $D$. With the help of (24) and (25) the value of $d^{2} I / d \alpha^{2}$ is found to be

$$
\frac{d^{2} I}{d \alpha^{2}}=\int_{s_{0}}^{s_{1}} w\left(f_{2} w-\frac{d}{d s} f_{1} w_{s}\right) d s+\left[\left(x_{a}^{2}+y_{a}^{2}\right)\left(\frac{P_{1}}{r}+P_{2}+Q \frac{w_{s}}{w}\right)\right]^{t=t_{1}},
$$

where

$$
\begin{aligned}
& P_{1}=-f \sin (\sigma-\tau)+f_{\tau} \cos (\sigma-\tau), \\
& P_{2}=\left(f_{x} \cos \sigma+f_{y} \sin \sigma\right) \cos (\sigma-\tau)+\left(f_{\tau_{x}} \cos \sigma+f_{\tau_{y}} \sin \sigma\right) \sin (\sigma-\tau) \\
& \begin{aligned}
Q=-f_{1} \sin ^{2}(\sigma-\tau) . & +f_{1} \sin (\sigma-\tau) \cos (\sigma-\tau) \tau_{x},
\end{aligned}
\end{aligned}
$$

But by means of the expression (26), the Jacobi condition can be proved * in the following form.

JACOBI'S NECESSARY CONDITION. If the arc $C$ is a solution of Euler's equation joining the fixed point 0 with the fixed curve $D$, and along which $f_{1} \neq 0$, then a further necessary condition for a minimum or a maximum is that no solution w of Jacobi's equation

exists which satisfies the condition

$$
f_{2} w-\frac{d}{d s} f_{1} w_{s}=0
$$

$$
\frac{P_{1}}{r}+P_{2}+Q \frac{w_{2}}{w}=0
$$

at the intersection of $C$ and $D$ and vanishes in the interval $s_{0}<s \leqq s_{1}$.

The alterations which must be made in the necessary conditions for the case of variable end-points in order to get sufficient conditions are so similar to the ones given in $\S 3$ that the corresponding theorem need not be stated here.

Princeton, March, 1907.

* See BLIss, The second variation of a definite integral when one end-point is variable, Transactions of the American Mathematical Society, vol. 3 (1902), p. 132. 